\title{
Facial Patterns are a Conventional Signal of Agonistic Ability in Polistes exclamans Paper Wasps
}

\author{
Elizabeth A. Tibbetts \& Michael J. Sheehan \\ Ecology and Evolutionary Biology, University of Michigan, Ann Arbor, MI, USA
}

\section{Correspondence}

Elizabeth A. Tibbetts, Ecology and

Evolutionary Biology, University of Michigan,

Ann Arbor, MI 48109, USA.

E-mail: tibbetts@umich.edu

Received: May 30, 2011

Initial acceptance: July 6, 2011

Final acceptence: September 12, 2011

(M. Hauber)

doi: $10.1111 / j .1439-0310.2011 .01967 . x$

\begin{abstract}
Some animals minimize the high costs of aggressive conflict by using conventional signals of agonistic ability to assess rivals prior to interacting. Conventional signals are more controversial than other signals of agonistic ability because they lack an inherent physical or physiological link with their bearer's agonistic ability. Here, we test whether the variable brown facial stripes in Polistes exclamans paper wasps function as a conventional signal. Polistes exclamans were given the option of challenging or avoiding a rival with an experimentally altered facial pattern. Our results show that rival assessment is based on the facial patterns of rivals, as well as an individual's own size, facial patterns, and nesting strategy. Individuals with larger body size and larger brown facial stripes were more likely to challenge rivals than individuals with smaller body size and smaller brown facial stripes. In addition, large individuals were more likely to challenge rivals with large brown facial stripes than small individuals, while an individual's own body size did not influence whether or not they challenged rivals with small brown stripes. Individuals who previously nested in multiple queen groups approached rivals more rapidly than individuals who previously nested alone, suggesting that social experience also plays a role in rival assessment. Finally, rivals with small facial stripes were challenged more rapidly than those with large facial stripes. These results demonstrate that $P$. exclamans facial patterns function as a signal used to minimize the cost of conflict. However, individuals do not make simple decisions based on their rival's signal alone, as an individual's own social experience and agonistic abilities also influence rival assessment decisions.
\end{abstract}

\section{Introduction}

Aggressive competition over limited resources is an important aspect of social interactions in many species. However, fights can be extremely costly, so individuals frequently assess the relative agonistic abilities of rivals before they engage in aggressive contests. Assessment reduces costly aggressive interactions by allowing individuals to avoid escalated contests with stronger or more motivated rivals (Maynard-Smith $\delta$ Harper 2003; Searcy \& Nowicki 2005).

In most taxa, rival assessment is based on signals that have an inherent association with their bearer's fighting ability (Parker 1974; Hurd 2006). For example, antlers convey information about fighting ability and are inherently linked to a male's ability to win a fight (Barrette \& Vandal 1990). In contrast, some taxa have conventional signals of fighting ability, traits that convey information about fighting ability, although there is no clear physical or physiological connection between the signal and its bearer's fighting ability (Guilford \& Dawkins 1995; Searcy \& Nowicki 2005). Conventional signals have been more controversial than other signals of fighting ability because they lack a required connection with their bearer's abilities (Senar 2006). As a result, it is 
less obvious how conventional signals could accurately convey information about fighting ability (Whitfield 1987). Nevertheless, there is experimental evidence that some species use visual conventional signals, or badges of status, to assess rivals (great tit, Parus major Jarvi \& Bakken 1984; harris sparrow, Zonotrichia querula Rohwer 1985; auklet, Aethia pusilla Jones 1990; siskin, Carduelis spinus Senar \& Camerino 1998; flat lizards, Platysaurus broadleyi Stapley \& Whiting 2006).

Signals of agonistic ability are expected to occur in systems where there is aggressive competition among numerous, unfamiliar individuals (Tibbetts $\delta$ Safran 2009). For example, Polistes dominulus paper wasps have variable facial patterns that function as conventional signals (Tibbetts $\&$ Lindsay 2008; Tibbetts et al. 2010), and a social system where nest-founding queens compete with many unfamiliar rivals (Roseler 1991). Aggressive competition is particularly important during the early nest-founding period. After paper wasp queens emerge from diapause, they spend approx. $2 \mathrm{wk}$ assessing potential nesting locations and nesting partners. Females typically battle with many rivals before starting a nest (Roseler 1991). The outcome of competition is important, as many individuals nest in cooperative groups with other unrelated females (Queller et al. 2000). Dominance rank within a group determines shares of reproduction, work, and access to food (West-Eberhard 1969; Strassmann \& Meyer 1983; Reeve 1991). Although the first few weeks of the nesting cycle are the most intense period of competition, competition with unfamiliar rivals continues to be important throughout the nest-founding period, as unfamiliar foundresses may usurp or join established nests (Nonacs \& Reeve 1995).

Here, we provide a new perspective on social signaling in the Polistes by testing the function of facial pattern variation in Polistes exclamans wasps. Testing the function of facial pattern variation in diverse paper wasps is important given the variation in social signaling within- and between-Polistes species (Tibbetts 2004). For example, within P. dominulus there is dramatic geographic variation in facial patterns, with some populations having high facial pattern variation and other populations having surprisingly low facial pattern variation (Cervo et al. 2008; Zanette \& Field 2009; E. A. Tibbetts, O. Skaldina, V. Zhao, A. L. Toth, M. Skaldin, L. Beani \& J. Dale, in review). In addition, there is variation in the importance of facial patterns during rival assessment across $P$. dominulus populations (Tibbetts et al. 2010; Green \& Field 2011). Across Polistes species, there are at least two different types of social signals. Polistes fuscatus have variable facial patterns that are used for individual recognition (Tibbetts 2002). Polistes dominulus have facial patterns that signal status (Tibbetts \& Lindsay 2008; Tibbetts et al. 2010). Polistes satan have facial patterns that predict status (Tannure-Nascimento et al. 2008), suggesting that they may function as status signals. In addition, there are multiple Polistes species with variable facial patterns with unknown signaling function (Rusina et al. 2007; Ortolani et al. 2010). Assessing the function of facial pattern variation across Polistes species will provide useful information about the generality of results obtained in previous work as well as the selective pressures that shape signal evolution.

We examine whether $P$. exclamans wasps have a conventional signal of fighting ability. P. exclamans are good candidates for this type of signal, as they have variable facial patterns (Fig. 1), and social behavior which involves competition among numerous, unfamiliar nest-founding queens (Strassmann 1983; Hughes \& Strassmann 1988; Willer \& Hermann 1989). First, we test whether variation in P. exclamans facial patterns is associated with body size. Body size is often associated with fighting ability and social status (Pardi 1948; but see Cant et al. 2006; Zanette $\&$ Field 2009), so a positive correlation between facial pattern and body size suggests that facial patterns could provide information about an individual's agonistic abilities. Second, we examine whether $P$. exclamans foundresses use facial patterns to assess rivals prior to interacting. Measuring whether facial patterns are used during rival assess-

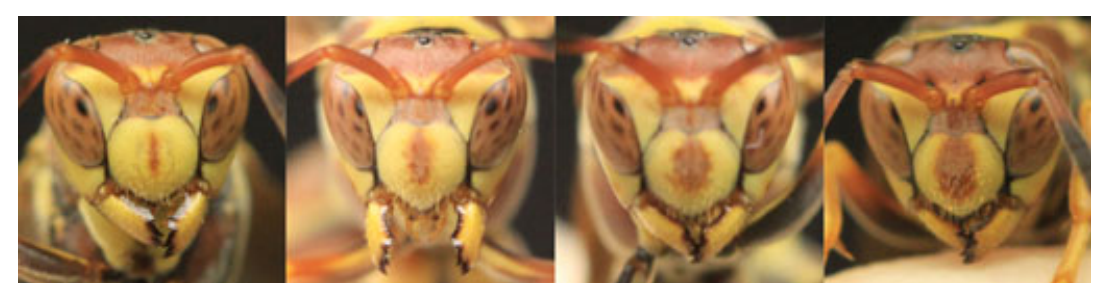

Fig. 1: Portraits of four Polistes exclamans foundresses, illustrating some of the variation in facial pattern. Individuals are arrayed from those signaling low agonistic ability (It) to those signaling high agonistic ability (rt). Head widths (It to rt: 3.68, 4.04, 3.96, 3.60 mm). 
ment provides a critical test of whether facial patterns function as a signal of fighting ability.

\section{Methods}

Polistes exclamans were collected from the wild near Austin, Texas USA $\left(30^{\prime} 15^{\circ} \mathrm{N}, 97^{\prime} 45^{\circ} \mathrm{W}\right)$ during the nest-founding stage from March 30 through April 5, 2010. The mean number of foundresses on a nest across all collection locations was 1.26 wasps per nests ( $\mathrm{n}=210$ nests, range $1-7$ foundress). Upon collection, each nest was individually housed with water and sugar. A random subset of foundresses were used in the choice trials.

Rival assessment decisions were tested by placing focal individuals in a trial arena that contained one patch of food guarded by another wasp. The focal wasps had the option of approaching the guard to gain access to food or avoiding the guarded food patch. Previous work in P. dominulus demonstrated that rival choice decisions are influenced by the facial pattern of the rival and the facial pattern of the individual making the decision (Tibbetts et al. 2010). Therefore, we tested how the guard and focal wasp characteristics influenced rival assessment during staged rival choice trials.

Choice trials were performed in a triangle-shaped arena $(7 \mathrm{~cm}$ wide $\times 6 \mathrm{~cm}$ long). There was a covered antechamber at the narrow end of the arena. At the other end of the arena, we placed a cube of sugar with a freshly killed guard wasp positioned on top. Each guard was freeze-killed and painted with brown and yellow paint to experimentally alter her facial patterns. The guards received a similar amount of the same type of paint in the clypeal region, although the appearance of the guards was altered in different ways. Two guards had their facial pattern increased with brown paint to produce guards with wider facial stripes that covered a larger fraction of the clypeus than their original facial stripes. In these guards, yellow paint was added over the yellow area of the clypeus as a control. Two guards had their facial pattern decreased with yellow paint to produce guards with narrower facial stripes that covered a smaller fraction of the clypeus than their original facial stripes. In these guards, brown paint was added over the brown area of the clypeus as a control. Multiple colors of Testors ${ }^{\mathrm{TM}}$ (Testors Co. Rockford, IL, USA) enamel paint were blended to produce a color that matched the guard's facial pattern. Guards originally had facial patterns around the population median and were altered, so they were in the upper or lower $15 \%$ of the population in terms of proportion of their clypeus pigmented brown. Relative guard facial pattern was assessed by photographing each wasp before and after facial pattern alteration measuring the resulting facial patterns in Adobe Photoshop, as described later. Guards were similarly sized (range $0.125-0.127 \mathrm{~g}$; population $\overline{\mathrm{x}}=0.120, \mathrm{SD}=0.028)$ and were randomly assigned to focal wasps. Guards and focal wasps were collected from sites at least $2 \mathrm{~km}$ apart to ensure they had not previously interacted.

Focal wasps were placed in the antechamber for $5 \mathrm{~min}$ before being released into the trial arena for $20 \mathrm{~min}$. All choice trials were videotaped, and behavior was later scored by an observer who was blind to experimental treatment and predictions. A wasp was considered to challenge the guard if she approached the guard and ate the sugar or climbed on top of the guard. Climbing on top of the guard is an aggressive behavior that appears similar to an attempted mount (West-Eberhard 1969). Most wasps that challenged the guard, both ate the sugar and climbed on top of the guard. Within wasps that challenged the guard, we measured the time between exiting the antechamber and challenging the guard (i.e. latency to challenge).

After behavioral experiments, wasps were freezekilled. Photographs of decapitated heads were later taken against a size standard. The facial pattern of each focal wasp was measured in ADOBE PHOTOSHOP CS3 (Adobe Systems, Inc. San Jose, CA, USA). For each wasp, we measured the width of the brown stripe at its widest point, the length of the stripe at its longest point, and the area of the brown stripe. Facial pattern parameters were size corrected for subsequent analyses: width brown was divided by the total clypeus width, length brown was divided by the total clypeus length, and area brown was divided by the total clypeus area. We also measured head width at the widest part of the face as a measure of body size. Head width provides a good method of overall structural size that is commonly used in research on Polistes (e.g. Zanette \& Field 2009).

Statistical analyses were performed using SPSS v. 17 (IBM, Armonk, NY, USA). The relationship between facial pattern and body size was tested using a regression. Whether or not the focal wasp challenged the guard was analyzed using generalized estimating equations. Individual guard identity was included as a subject variable in the statistical analysis to control for possible similarity in response to the same guard. This is important because it controls for individual-specific variation among guards that 
could influence focal wasp choices. The dependent variable was whether or not the focal wasp challenged the guard (i.e. ate the sugar or sat on the guard). Latency to eat was analyzed using a mixed linear model with specific guard as a random effect to control for possible similarity in response to the same guard. For both analyses, the independent variables included: focal wasp structural size (head width), focal wasp facial pattern, focal wasp nesting strategy (collected from a single foundress or multiple foundress nest), and guard facial pattern (experimentally increased to large brown stripe vs. experimentally decreased to small brown stripe). All two way interactions were included in the original model. Non-significant interactions were removed from the final model; though, they are also reported. One hundred and thirteen choice trials were performed using four different guards.

\section{Results}

The brown clypeus patterns in P. exclamans vary dramatically in relative width and proportion brown, while length is less variable (relative width $\mathrm{CV}=0.52$, proportion brown $\mathrm{CV}=0.41$, relative length $\mathrm{CV}=0.12$ ). Therefore, we focus on proportion brown and relative width in subsequent analyses. Both relative width and proportion brown are correlated with individual head width (Fig. 2, proportion brown, $F_{1,111}=17.1, \mathrm{p}<0.001, r^{2}=0.13$; relative width, $\left.F_{1,111}=8.7, \mathrm{p}=0.004, r^{2}=0.07\right)$. Larger individuals have wider facial stripes that cover a larger fraction of their clypeus. Not surprisingly, the relative width of the brown strip and the proportion of the clypeus pigmented brown are highly, positively correlated (relative width vs. proportion brown $F_{1,111}=611.6, \mathrm{p}<0.001, r^{2}=0.85$ ).

The proportion of the clypeus pigmented brown is more strongly associated with head width than the relative width of the brown clypeus stripe. As a result, the choice trial analyses presented later use proportion of the clypeus pigmented brown as the key facial pattern parameter. Analyses using the relative width of the brown stripe yield similar results.

Individual decisions about whether or not to challenge a guard were influenced by the facial pattern of the guard as well as the focal wasp's own characteristics. Whether or not the focal wasp challenged the guard was influenced by the guard's facial pattern (Wald $\chi^{2}=21.9, \mathrm{p}<0.001$ ), focal wasp facial pattern (proportion brown, Wald $\chi^{2}=6.2, \mathrm{p}=$ 0.012 ), focal wasp head width (Wald $\chi^{2}=8.3$, $p=0.004$ ), focal wasp nesting behavior (single or
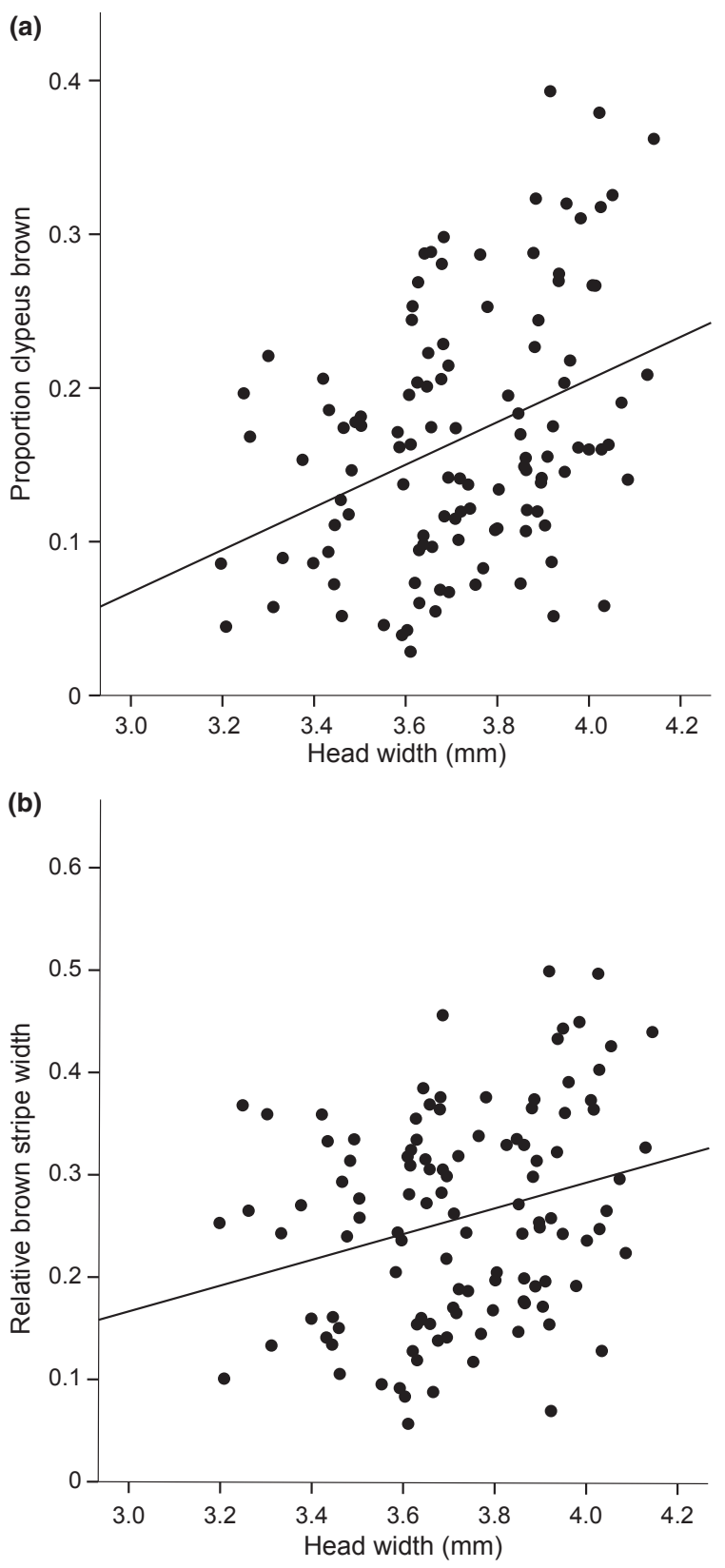

Fig. 2: (a) Relationship between head width and fraction of the clypeus pigmented brown. $\left(r^{2}=0.13\right)$. (b) Relationship between head width and the width of the brown clypeus stripe at its widest (measured as proportion of the clypeus width brown) $\left(r^{2}=0.07\right)$.

multiple foundress, Wald $\left.\chi^{2}=19.8, \mathrm{p}<0.001\right)$, the interaction between guard facial pattern and focal wasp head width (Wald $\chi^{2}=22.7, \mathrm{p}<0.001$ ), the interaction between focal wasp facial pattern and head width (Wald $\chi^{2}=8.8, p=0.003$ ), and the interaction between focal wasp nesting behavior and facial pattern (Wald $\chi^{2}=24.5, \mathrm{p}<0.001$ ), but not the interaction between guard facial pattern and 
focal wasp facial pattern (Wald $\chi^{2}=0.43, p=0.51$ ), the interaction between focal wasp nesting behavior and head width (Wald $\chi^{2}=1.2, \mathrm{p}=0.26$ ), or the interaction between guard facial pattern and focal wasp nesting behavior (Wald $\chi^{2}=2.3, \mathrm{p}=0.12$ ). Individuals who challenged guards with large brown facial stripes had a larger head width and a larger fraction of their clypeus pigmented brown than those that challenged guards with smaller brown facial stripes. In addition, large individuals were more likely to challenge guards with large brown stripes than small individuals, while head width did not influence whether or not individuals challenged guards with small brown stripes (Fig. 3). Finally, wasps from multiple foundress nests were more likely to challenge the guard than wasps from single foundress nests.

Latency to challenge the guard was influenced by guard facial pattern $\left(F_{1,73}=6.0, \mathrm{p}=0.017\right)$ and whether the focal wasp was from a single or multiple foundress nest $\left(F_{1,74}=7.02, \mathrm{p}=0.01\right)$. Focal wasps challenged guards with smaller brown stripes more rapidly than guards with larger brown stripes (Fig. 4). Wasps from multiple foundress nests challenged the guard more rapidly than wasps from single foundress nests (Fig. 4). Other focal wasp characteristics did not influence latency to challenge the guard (focal wasp

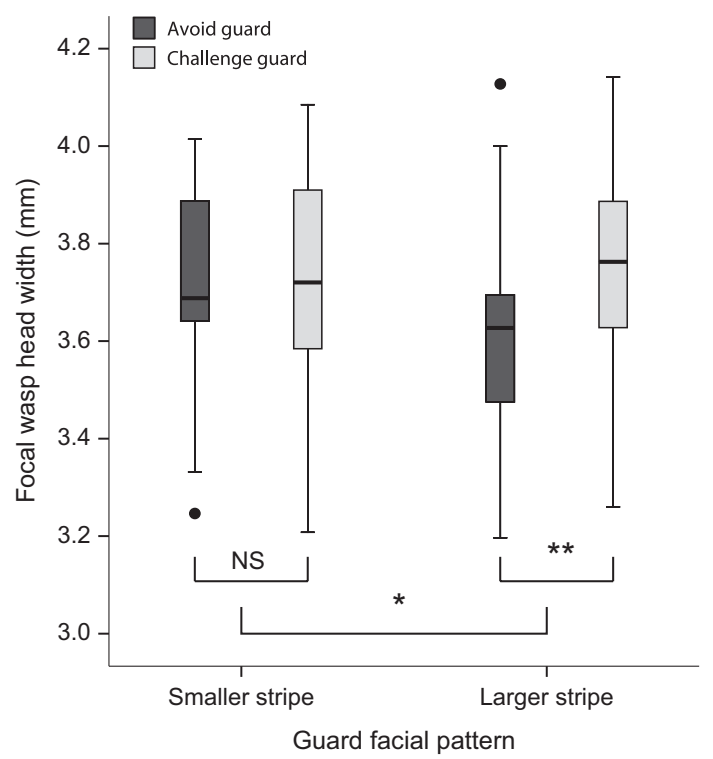

Fig. 3: The head width of focal wasps that decided to challenge (light gray) vs. avoid (dark gray) rivals with large vs. small brown facial stripes. Box indicates the 25th, 50th, and 75th percentile, and the whiskers reflect the minimum and maximum (except for the two dots, which reflect extreme data points that are more than three inter-quartile ranges from the end of the box) ( $\left.{ }^{\star} p<0.05,{ }^{*} p<0.001\right)$.

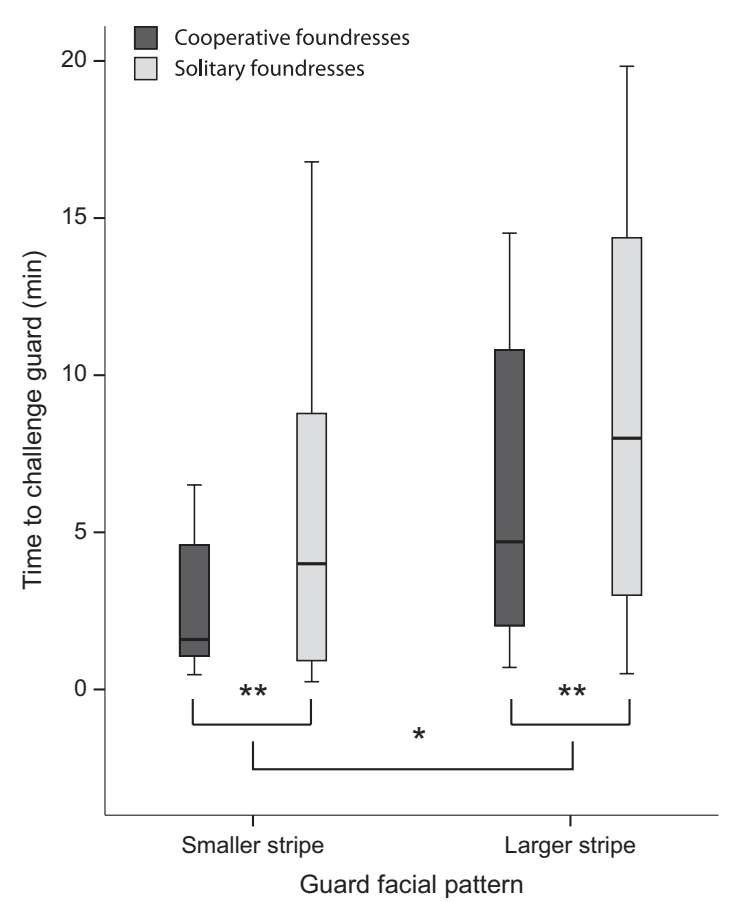

Fig. 4: The latency to challenge guards with large vs. small brown facial stripes. Focal wasps challenged guards with small brown stripes more rapidly than guards with large brown stripes $\left({ }^{*} p<0.05\right.$, $* * p<0.001)$. Box indicates the 25th, 50th, and 75th percentile, while the whiskers reflect the minimum and maximum.

head width, $F_{1,73}=0.21, \mathrm{p}=0.65$; focal wasp facial pattern, $\left.F_{1,73}=0.17, \mathrm{p}=0.68\right)$. In addition, none of the interactions significantly influenced latency to challenge (guard facial pattern by focal wasp head width $F_{1,67}=0.34, \mathrm{p}=0.57$; guard facial pattern by focal wasp facial pattern $F_{1,67}<0.01, \mathrm{p}=0.95$; focal wasp head width by focal wasp facial pattern $F_{1,67}=0.07, \mathrm{p}=0.79$; focal wasp nesting behavior by guard facial pattern $F_{1,67}<0.01, \mathrm{p}=0.96$; focal wasp nesting behavior by head width $F_{1,67}=1.9, \mathrm{p}=0.17$; or focal wasp nesting behavior by facial pattern $\left.F_{1,67}=0.87, \mathrm{p}=0.35\right)$.

\section{Discussion}

Polistes exclamans have variable facial patterns that function as a conventional signal of agonistic ability. Wasps with a higher proportion of their clypeus pigmented brown are larger than wasps with a smaller proportion of their clypeus pigmented brown (Fig. 2). These facial patterns are used during rival assessment; foundresses decide whether or not to challenge a rival based on the facial pattern of the rival as well as their own body size and facial pattern. 
Instead of using a simple decision rule based on rival quality alone, $P$. exclamans foundresses incorporate information about their own and rival ability during assessment (Fig. 3). Larger P. exclamans or individuals with more brown on their clypeus are more willing to challenge rivals than smaller $P$. exclamans or individuals with less brown on their clypeus. There are persistent questions about how accurately signals reflect their bearer's abilities, and many studies have found weak relationships between signal phenotype and fighting ability (Bradbury \& Vehrencamp 2000; Taylor \& Elwood 2003). Therefore, it is particularly interesting that $P$. exclamans facial patterns are linked to their bearer's contest behavior; facial patterns provide valuable information about their bearer's behavioral strategy. Interestingly, body size and facial pattern are independently associated with behavioral strategy, suggesting that these traits provide different information about agonistic behavior to rivals. Future experiments will be important to tease apart the relative roles of size and facial pattern during competition. In addition, experiments using guards with varying body size will be essential to test whether body size alone functions as a signal or cue of fighting ability. The relationship between focal wasp size and behavior in this experiment suggests that body size plays a role during aggressive competition. However, guard size was standardized across trials because this study was designed to test the role of facial patterns during rival assessment. As a result, this study does not explicitly test whether wasps use body size to assess rivals.

The focal wasp's nest-founding strategy was also associated with rival challenge behavior; solitary queens were less likely to challenge guards and had a longer latency to challenge than cooperative queens. These results suggest that nesting strategy is associated with broad differences in social behavior, with cooperative individuals showing a greater willingness to approach conspecifics than solitary individuals. These behavioral differences could be a cause or a consequence of cooperation; cooperation may change an individual's willingness to engage conspecifics or there may be pre-existing individual differences that influence the formation of cooperative associations. Although there has been extensive research on the evolution of cooperation (reviewed in Lehmann \& Keller 2006), surprisingly little is known about the causes and consequences of cooperation at the individual level (reviewed in Bergmuller et al. 2010). Future research that tests for consistent individual variation in cooperativeness will be useful to understand whether there are indi- viduals with 'cooperative personalities (behavioral syndromes)' and the implications behavioral syndromes for the evolution of cooperation (Bergmuller et al. 2010).

The conventional signal in P. exclamans likely minimizes the costs of conflict during dominance competition among nest-founding queens. Conventional signals are expected to be particularly valuable for assessing unfamiliar rivals (Rohwer 1975; Tibbetts \& Safran 2009), so wasps may primarily use visual signals during the early nest-founding phase when potential queens battle for dominance, colony membership is flexible and nest usurpation is common (Roseler 1991; Nonacs \& Reeve 1995). Conventional signals are less important within stable groups (Vedder et al. 2010), so facial patterns may be less important during communication within nests. Instead, wasps may use cuticular hydrocarbons that are well known signals of fertility (Monnin 2006) to mediate interactions on stable nests.

Previous work on conventional signals of agonistic ability in Polistes paper wasps has focused on P. dominulus. Interestingly, there are remarkable similarities between experimental results in $P$. dominulus and P. exclamans; though, the species are distantly related (Carpenter 1996; Pickett et al. 2006). In both systems, facial patterns are weakly but significantly associated with body size (Tibbetts \& Dale 2004; Tibbetts 2006 but see Tibbetts \& Curtis 2007; Cervo et al. 2008). Body size is commonly associated with fighting ability (Pardi 1948; but see Cant et al. 2006), so the relationship between signal and body size suggests that the signals can convey information about fighting ability. Also, foundresses in both species use mutual rival assessment, as they incorporate information about their own quality and a rival's quality during signal assessment (this study, Tibbetts et al. 2010). In both systems, it is unclear how individuals acquire information about their own quality; experience and/or physiological state may both play important roles. Future work will be useful to establish whether the independently evolved agonistic signal in $P$. exclamans has other parallels with $P$. dominulus. For example, is signal development condition-dependent (Tibbetts 2010)? Do social costs play a role in maintaining the accuracy of $P$. exclamans facial patterns (Tibbetts \& Izzo 2010)?

Although there are substantial similarities in the signaling systems of $P$. dominulus and P. exclamans, there are also some differences. First, there are striking differences in the type of facial pattern variation. Polistes dominulus have extremely broken or wavy black facial spots, and brokenness is the 
aspect of facial patterns that conveys information about fighting ability (Tibbetts \& Dale 2004). In contrast, P. exclamans have smooth facial spots that vary in width and area rather than brokenness. Independently evolved signaling systems often have very different properties (Bradbury \& Vehrencamp 1998), so the variation in facial patterns across species isn't particularly surprising, but the variation does suggest that future comparative work on signal development across species will be quite interesting. Another difference between the two species is that body size and nesting strategy are important factors influencing P. exclamans rival assessment behavior, while neither factor has been included in previous $P$. dominulus rival assessment studies (Tibbetts et al. 2010; Green \& Field 2011). Size is inconsistently related to dominance across populations of the same Polistes species (Pardi 1948; Cant et al. 2006; Zanette $\delta$ Field 2009), so variation in the role of size across species would not be surprising. However, the variation suggests future models should include multiple factors to provide a full picture of rival assessment.

Most Polistes species are thought to lack visual signals; though, the type of variable facial patterns used for social communication has evolved multiple times across the genus (Tibbetts 2004). Polistes dominulus, P. satan, and P. exclamans have facial patterns that signal agonistic ability (this study, Tannure-Nascimento et al. 2008; Tibbetts et al. 2010). All three species have continuous variation in clypeus coloration; though, the type of clypeus coloration that signals agonistic ability varies across species: brokenness of black spots in P. dominulus, proportion of brown vs. black in P. satan, and proportion brown vs. yellow in P. exclamans. Another paper wasp species, P. fuscatus, has facial patterns that signal individual identity (Tibbetts 2002; Sheehan \& Tibbetts 2009). Polistes fuscatus have much more variable facial pattern than any of the species with quality signals, as $P$. fuscatus have variable black, brown, and yellow coloration in the inner eye, eyebrow, outer eye, clypeus, and abdomen (Sheehan \& Tibbetts 2010). The higher variation in P. fuscatus than the three species with quality signals is consistent with the prediction that identity signals are more variable than quality signals (Dale 2000; Tibbetts \& Dale 2007). Two additional Polistes species have pronounced intra-population variation in facial patterns, P. nimpha (Rusina et al. 2007) and P. sulcifer (Ortolani et al. 2010), suggesting that these species may also have visual signals. Future studies on social signaling across the Polistes will be useful to test how frequently and under what circumstances different types of visual signals evolve.

Overall, P. exclamans paper wasps have variable facial patterns that function as signals of agonistic ability. Polistes exclamans use mutual assessment during contests, as rival choice decisions are influenced by an individual's own size, facial patterns, and nesting strategy as well as the facial pattern of rivals. Our results illustrate the importance of considering an individual's own quality during analysis of communication experiments. In addition, they add to a growing body of research suggesting that visual signals of agonistic ability may be widespread in the paper wasps.

\section{Acknowledgements}

We thank Kellie Donajkowski, Jessica Chovanec, Alex Mettler, Eren Siphai, Brittany Willingham, and Sally Wingard for research assistance. Funding was provided by the University of Michigan.

\section{Literature Cited}

Barrette, C. \& Vandal, D. 1990: Sparring, relative antler size, and assessment in male caribou. Behav. Ecol. Sociobiol. 26, 383-387.

Bergmuller, R., Schurch, R. \& Hamilton, I. M. 2010 : Evolutionary causes and consequences of consistent individual variation in cooperative behaviour. Philos. Trans. R. Soc. Lond., B, Biol. Sci. 365, 2751-2764.

Bradbury, J. \& Vehrencamp, S. 1998: Principles of Animal Communication. Sinauer Associates, Sunderland, MA.

Bradbury, J. W. \& Vehrencamp, S. L. 2000: Economic models of animal communication. Anim. Behav. 59, $259-268$.

Cant, M. A., English, S., Reeve, H. K. \& Field, J. 2006: Escalated conflict in a social hierarchy. Proc. Biol. Sci. 273, 2977-2984.

Carpenter, J. M. 1996: Phylogeny and biogeography of Polistes. In: Natural History and Evolution of Paper Wasps (Turillazzi, S. \& West-Eberhard, M. J., eds). Oxford Univ. Press, New York, NY, pp. 18-57.

Cervo, R., Dapporto, L., Beani, L., Strassmann, J. E. \& Turillazzi, S. 2008: On status badges and quality signals in the paper wasp Polistes dominulus: body size, facial colour patterns and hierarchical rank. Proc. Biol. Sci. 275, 1189-1196.

Dale, J. 2000: Ornamental plumage does not signal male quality in red-billed queleas. Philos. Trans. R. Soc. Lond., B, Biol. Sci. 267, 2143-2149.

Green, J. P. \& Field, J. 2011: Interpopulation variation in status signalling in the paper wasp Polistes dominulus. Anim. Behav. 81, 205-209. 
Guilford, T. \& Dawkins, M. S. 1995: What are conventional signals? Anim. Behav. 49, 1689-1695.

Hughes, C. R. \& Strassmann, J. E. 1988: Foundress mortality after worker emergence in social wasps (Polistes). Ethology 79, 265-280.

Hurd, P. L. 2006: Resource holding potential, subjective resource value, and game theoretical models of aggressiveness signalling. J. Theor. Biol. 241, 639-648.

Jarvi, T. \& Bakken, M. 1984: The function of the variation in the breast stripe of the great tit Parus major. Anim. Behav. 32, 590-596.

Jones, I. L. 1990: Plumage variability functions for status signalling in least auklets. Anim. Behav. 39, 967-975.

Lehmann, L. \& Keller, L. 2006: The evolution of cooperation and altruism-a general framework and a classification of models. J. Evol. Biol. 19, 1365-1376.

Maynard-Smith, J. \& Harper, D. 2003: Animal Signals. Oxford Univ. Press, New York, NY.

Monnin, T. 2006: Chemical recognition of reproductive status in social insects. Ann. Zool. Fenn. 43, 515-530.

Nonacs, P. \& Reeve, H. K. 1995: The ecology of cooperation in wasps - causes and consequences of alternative reproductive decisions. Ecology 76, 953-967.

Ortolani, I., Zechini, L., Turillazzi, S. \& Cervo, R. 2010 : Recognition of a paper wasp social parasite by its host: evidence for a visual signal reducing host aggressiveness. Anim. Behav. 80, 683-688.

Pardi, L. 1948: Dominance order in Polistes wasps. Physiol. Zool. 21, 1-13.

Parker, G. A. 1974: Assessment strategy and evolution of fighting behavior. J. Theor. Biol. 47, 223-243.

Pickett, K. M., Carpenter, J. M. \& Wheeler, W. C. 2006: Systematics of Polistes (Hymenoptera : Vespidae), with a phylogenetic consideration of Hamilton's haplodiploidy hypothesis. Ann. Zool. Fenn. 43, 390-406.

Queller, D. C., Zacchi, F., Cervo, R., Turillazzi, S., Henshaw, M. T., Santorelli, L. A. \& Strassmann, J. E. 2000: Unrelated helpers in a social insect. Nature 405 , 784-787.

Reeve, H. K. 1991: Polistes. In: The Social Biology of Wasps (Ross, K. G. \& Matthews, R. W., eds). Comstock Publishing Associates, Ithaca, pp. 99-148.

Rohwer, S. 1975: The social significance of avian winter plumage variability. Evolution 29, 593-610.

Rohwer, S. 1985: Dyed birds achieve higher social status than controls In Harris Sparrows. Anim. Behav. 33, 1325-1331.

Roseler, P. F. 1991: Reproductive competition during colony establishment. In: The social biology of wasps (Ross, K. G. \& Matthews, R. W., eds). Comstock publishing associates, London, pp. 309-335.

Rusina, L. Y., Rusin, I. Y., Starr, C. K., Fateryga, A. B. \& Firman, L. A. 2007: Modes of colony foundation by females of different morphotypes in paper wasps
(Hymenoptera, Vespidae, Polistes Latr.). Entomol. Rev. 87, 1155-1173.

Searcy, W. A. \& Nowicki, S. 2005: The Evolution of Animal Communication. Princeton Univ. Press, Princeton.

Senar, J. C. 2006: Color displays as intrasexual signals of aggression and dominance. In: Bird Coloration Function; and Evolution (Hill, G. E. \& McGraw, K. J., eds). Harvard Univ. Press, London, pp. 87-136.

Senar, J. C. \& Camerino, M. 1998: Status signalling and the ability to recognize dominants: an experiment with siskins (Carduelis spinus). Philos. Trans. R. Soc. Lond., B, Biol. Sci. 265, 1515-1520.

Sheehan, M. J. \& Tibbetts, E. A. 2009: Evolution of identity signals: frequency-dependent denefits of distinctive phenotypes used for individual recognition. Evolution 63, 3106-3113.

Sheehan, M. J. \& Tibbetts, E. A. 2010: Selection for individual recognition and the evolution of polymorphic identity signals in Polistes paper wasps. J. Evol. Biol. 23, 570-577.

Stapley, J. \& Whiting, M. 2006: Ultraviolet signals fighting ability in a lizard. Biol. Lett. 2, 169-170.

Strassmann, J. E. 1983: Nest fidelity and group size among foundresses of Polistes annularis (Hymenoptera, Vespidae). J. Kans. Entomol. Soc. 56, 621-634.

Strassmann, J. E. \& Meyer, D. C. 1983: Gerontocracy in the social wasp, Polistes exclamans. Anim. Behav. 31, 431-438.

Tannure-Nascimento, I. C., Nascimento, F. S. \& Zucchi, R. 2008: The look of royalty: visual and odour signals of reproductive status in a paper wasp. Proc. Biol. Sci. 275, 2555-2561.

Taylor, P. W. \& Elwood, R. W. 2003: The mismeasure of animal contests. Anim. Behav. 65, 1195-1202.

Tibbetts, E. A. 2002: Visual signals of individual identity in the wasp Polistes fuscatus. Proc. Biol. Sci. 269, $1423-1428$.

Tibbetts, E. A. 2004: Complex social behaviour can select for variability in visual features: a case study in Polistes wasps. Proc. Biol. Sci. 271, 1955-1960.

Tibbetts, E. A. 2006: Badges-of-status in worker and gyne Polistes dominulus wasps. Ann. Zool. Fenn. 43, 575-582.

Tibbetts, E. A. 2010: The condition-dependence and heritability of signaling and non-signaling color traits in paper wasps. Am. Nat. 175, 495-503.

Tibbetts, E. A. \& Curtis, T. R. 2007: Rearing conditions influence quality signals but not individual identity signals in Polistes wasps. Behav. Ecol. 18, 602-607.

Tibbetts, E. A. \& Dale, J. 2004: A socially enforced signal of quality in a paper wasp. Nature 432, 218-222.

Tibbetts, E. A. \& Dale, J. 2007: Individual recognition: it is good to be different. Trends Ecol. Evol. 22, $529-537$. 
Tibbetts, E. A. \& Izzo, M. 2010: Social punishment of dishonest signalers caused by mismatch between signal and behavior. Curr. Biol. 20, 1637-1640.

Tibbetts, E. A. \& Lindsay, R. 2008: Visual signals of status and rival assessment in Polistes dominulus paper wasps. Biol. Lett. 4, 237-239.

Tibbetts, E. A. \& Safran, R. J. 2009: Co-evolution of black plumage patches and winter sociality in new and old world sparrows. J. Evol. Biol. 22, 2376-2386.

Tibbetts, E. A., Mettler, A. \& Levey, S. 2010: Mutual assessment via visual status signals in Polistes dominulus wasps. Biol. Lett. 6, 10-13.

Vedder, O., Schut, E., Magrath, M. J. L. \& Komdeur, J. 2010: Ultraviolet crown colouration affects contest out- comes among male blue tits, but only in the absence of prior encounters. Funct. Ecol. 24, 417-425.

West-Eberhard, M. 1969: The social biology of polistine wasps. Misc. Publ., Mus. Zool., Univ. Mich. 140, 1-101. Whitfield, D. P. 1987: Plumage variability, status signaling and individual recognition in avian flocks. Trends Ecol. Evol. 2, 13-18.

Willer, D. E. \& Hermann, H. R. 1989: Multiple foundress associations and nest wwitching among females of Polistes exclamans (Hymenoptera, Vespidae). Sociobiology 16, 197-216.

Zanette, L. \& Field, J. 2009: Cues, concessions and inheritance: dominance hierarchies in the paper wasp Polistes dominulus. Behav. Ecol. 20, 773-780. 